This item was submitted to Loughborough's Research Repository by the author.

Items in Figshare are protected by copyright, with all rights reserved, unless otherwise indicated.

\title{
Motion-control techniques of today and tomorrow: A review and discussion of the challenges of controlled motion
}

PLEASE CITE THE PUBLISHED VERSION

https://doi.org/10.1109/MIE.2019.2956683

\section{PUBLISHER}

leee

VERSION

AM (Accepted Manuscript)

\section{PUBLISHER STATEMENT}

Personal use of this material is permitted. Permission from IEEE must be obtained for all other uses, in any current or future media, including reprinting/republishing this material for advertising or promotional purposes, creating new collective works, for resale or redistribution to servers or lists, or reuse of any copyrighted component of this work in other works.

\section{LICENCE}

\section{All Rights Reserved}

\section{REPOSITORY RECORD}

Ruderman, M, M Iwasaki, and Wen-Hua Chen. 2020. "Motion-control Techniques of Today and Tomorrow: A Review and Discussion of the Challenges of Controlled Motion". Loughborough University. https://hdl.handle.net/2134/14047004.v1. 


\title{
Motion control techniques working today and appealing tomorrow
}

\section{Reviewing and discussing challenges of controlled motion}

\author{
Michael Ruderman, Makoto Iwasaki, Wen-Hua Chen
}

Motion control technologies are in core of multiple mechatronic products and applications. Wherever an actuated motion takes place in machines and components, either position or force setting (correspondingly trajectory tracking), or even a combination of both, are demanded from the control system. In high-performance mechatronic systems including micro- and/or nano-scale motion (such as data storage devices, machine tools, manufacturing tools for electronics components, and industrial robots), the required specifications in motion performance, e.g., response/settling time and trajectory/settling accuracy, should be sufficiently achieved [1]. From an industrial perspective, the most common design objective for advanced motion control is to achieve a good tracking/following performance in the presence of disturbances and modeling issues - nominal plant and performance specifications [2]. While motion control, as an applied field of control theory, established itself already in nineties [3], [4], being also independently driven by the contemporary research in robotics [5], an incessant progress in the integrated mechatronic design and novel actuator technologies set new challenges for motion control technologies. Likewise, the today's requirements on material- and energy-saving push the mechatronic systems towards sensor and actuator reduction, hence feedback degradation and underactuation, equally as towards more lightweight and therefore flexible (to say soft) structures. These trends burden a robust and efficient motion control with some ineluctable theoretical and practical issues of different nature. Among those are the plant, specifications, and disturbance modeling, variable structure systems, identification and state estimation, control robustness and adaptivity, and others.

In this article, we partially review the state-of-the-art and discuss some recent and potential challenges of the motion control techniques and related developments. Our goal is to highlight and bring to attention of the interested auditorium of IEEE Industrial Electronics Society (IES) the principal features and associated issues of the controlled motion in advanced mechatronic systems and applications. By doing it, we are referring to the works published not only in IES journals and conferences but, rather, associated with a broadly spread research community around the motion control topics and affiliated technologies. Starting with basic principles of the motion stiffness and ideal versus disturbed motion under control, we clarify the vibrational perturbations owing to the structures, and damping perturbations owing to the friction on contact interfaces. The motion stiffness is introduced in Section I and that for an ideal position and force control, following by the mechanical impedance control as a more general case. In Section II we put an ideal versus disturbed motion under control, with associated canonical form of the state-space system modeling. Vibrational and frictional perturbations, as often most pronounced in the controlled motion systems, are highlighted in Sections III and IV correspondingly. Next, we discuss the issues of measurement and observation of dynamic states in the motion systems, before referring to some related motion control and compensation techniques. Section V explains the problem of measuring the process variables and approaches the state-of-the-art of estimation techniques. Control and compensation

M. Ruderman is with Department of Engineering Sciences, University of Agder, Grimstad, Norway; (e-mail: michael.ruderman@uia.no). M. Iwasaki is with Department of Mechanical and Electrical Engineering, Nagoya Institute of Technology, Nagoya, Japan; (e-mail: iwasaki@nitech.ac.jp). W.-H. Chen is with Department of Aeronautical and Automotive Engineering Loughborough University, Loughborough, U.K.; (e-mail: w.chen@lboro.ac.uk). 
strategies for the matched and mismatched disturbances are summarized in Section VI. We draw some basic conclusions by the end of the article in Section VII.

\section{I. "STIFFNESS" OF MOTION: FROM POSITION TO FORCE CONTROL}

In control engineering practice, the so-called "stiffness" is often used in everyday language, in order to characterize the bandwidth of closed-loop control system, correspondingly the time constants of its transient response. In that regard, a "stiff" control loop provides faster response times and its corner, correspondingly bandwidth, frequencies lie further to the right, comparing to a less "stiff" control system. This appears in direct analogy to the basic second-order mechanical systems with mass-spring-damper elements, for which the stiffness of restoring spring determines the natural frequency and, therefore, dynamic characteristics of the system response.

While the term stiffness sounds like something self-evident for mechanical structures and, in equivalence to the second-order dynamics, for closed-loop control systems as well, the so-called "stiffness" of controlled motion needs first to be clarified for convenience of the further use. From the point of view of force balance, a controlled motion (here with one degree of freedom for the sake of simplicity) can be seen as a generic (nonlinear) map

$$
F=h(X, t)
$$

between the generalized force $F$, totally imposed on the motion system, and the resulted relative displacement expressed in the generalized coordinates $X$. Implicitly, the relation (1) incorporates both - the motion plant and motion control system - and is function of equally the time derivatives of displacement (of a necessary order). The stiffness of the controlled motion can be then defined, cf. e.g. [3], as a partial derivative

$$
K=\frac{\partial F}{\partial X}
$$

It is worth noting that the operation point of a generally nonlinear plant, equally as more advanced nonlinear (often hybrid and/or adaptive) controllers, can lead to a time-varying stiffness, thus resulting in $K(t)$. The concept of stiffness of the controlled motion has been used in [3], [6] as particularly suitable for a uniform consideration of the position (or velocity) and force control, equally as different combinations of both. A high robustness of the motion system brings the adjustable stiffness and, as a result, versatile applications will be possible [6]. One should notice that, quite often, to satisfy varying requirements of the motion control application at hand, the controller should have variable stiffness while keeping the system highly robust; cf. Figure 1 which conceptualizes the motion stiffness for different control cases.

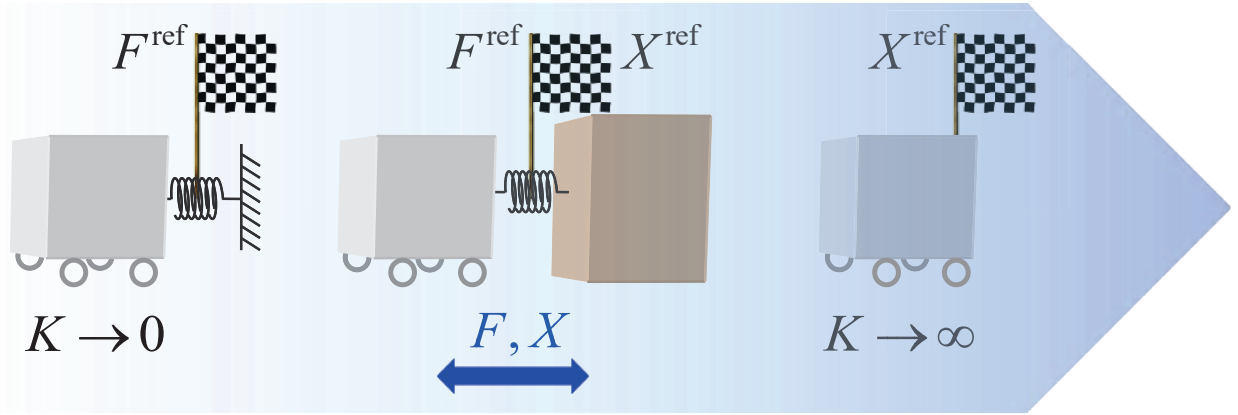

Fig. 1. Stiffness of controlled motion for the force (left), impedance (middle), and position (right) control. The motion stiffness increases from the left to the right, while the flag represents the reference set value.

For designing an ideal position, or alternatively velocity, control one has to ensure the controlled motion system is unsensitive to variation of the forces, which can appear as weakly known and rather unpredictable disturbances. That means for any deviations $\partial F$, an ideal position control inhibits any deviations in the desired position i.e. $\partial X \rightarrow 0$, that implies an infinite stiffness $K \rightarrow \infty$. This is also in accordance with 
the internal model principle [7], which for example requires an integral control action to be incorporated in order to compensate for the constant force disturbances so that $\partial X \rightarrow 0$ as $t \rightarrow \infty$. Similar as an high-stiffness mechanical structure will deform less in response to the applied force, that is beneficial for high precision and thus position accuracy, a stiff motion control design will ensure an insensitive operation in the presence of external force disturbances. While an infinite static stiffness is provided by the integral-of-position-error state feedback, the sometimes called dynamic stiffness [8] should take into account more specific behavior of the foresighted disturbances. Note that the static and dynamic stiffness terms, used in [8], refer to infinitely high steady-state stiffness, due to the integral loop characteristics, and adjustable stiffness scalable for a particular frequency range of disturbance. This requires mostly a more elaborated and often adaptive control design. However, such control flexibility has usually to make a tradeoff between some specific disturbance characteristics, correspondingly modeling, and robust performance. The latter means for a broader amplitude and frequency range of the exogenous signals, i.e. reference trajectories and disturbances. Once the relative displacement state is available and used for control, a robust controller with high-gain feedback renders the motion stiffness to be respectively high. Therefore, with an appropriately shaped closed-loop damping, the infinite-gain feedback design can approach theoretically an ideal position control with the desired infinite stiffness. At the same time, the inherent actuator constraints make this theoretical realization impossible for practical applications, since already the high-gain control actions, including also an integral-of-position-error feedback, may hit the limits of the saturated actuator elements. Here it is worth noting that a robust, also in terms of performance robustness, tracking controller with an explicit disturbance compensator (as addressed later in Section VI) does not necessarily require an integral control action. This has been shown already in [4] for a high-accuracy positioning system and explicitly analyzed in [9] for the nonlinear frictional-type disturbances.

In the same spirit of stiffness of the controlled motion, cf. (2), an ideal force control should allow infinite deviation of position, i.e. $\partial X \rightarrow \infty$, except the case that the force error is completely zero, cf. [6]. This implies the control stiffness tends to be zero, i.e. $K \rightarrow 0$, under nominal conditions of the force regulation. From that point of view, an ideal force controller should drive the system to $\partial F \rightarrow 0$, and that through an induced relative motion which will minimize the force error. That means to keep the total system force on some desirable balance value $F(t)=$ const. One should notice that unlike a pure position control, the force control requires distinguishing between two principally different modes: (i) an unconstrained non-contact motion and (ii) contact motion constrained by the system environment. In the non-contact mode, a force control is substantially an acceleration control, cf. [3]. Here we recall that the input of robust motion controller should be the acceleration quantity, cf. [6]. Therefore, for realizing versatile motion control systems, whose control stiffness can widely change, the overall motion controller should have a doublecascade structure. The inner robust (against disturbances) acceleration loop is for accurately tracking the desired acceleration reference. The outer loop has function of adjustment of the motion stiffness, by generating an appropriate acceleration reference. It is worth noting that this principle, exposed in [6], has been independently developed also in the robotic research [5], [10], [11], and since then accommodated in standard robotic literature. It is also well-known as feedback linearization, which is often denoted (in robotics) as an inverse dynamics control or computed torque control. The latter namely transmits the coupled nonlinear manipulator dynamics into well-known double integrator system, cf. e.g. [12]. An acceleration control, correspondingly force control, during non-contact motion contains mainly a forward gain, which scales the force reference to an acceleration quantity, provided the system inertia is known. Except the less common applications, where an exact acceleration measurement is available for feedback, a force control has no feedback loops during the non-contact motion. This appears quite naturally since no contact with environment does not return any force sensing from the tactile (end-effector) interface. But once the forced-controlled motion system comes in touch with environment, a closed mechanical loop arises through the environmental stiffness and damping which become the principal factors in the dynamics of a force-controlled system. With typically insufficient and not ideally viscous environmental damping, the force-controlled motion system becomes oscillatory at contact and, in consequence, is repulsed back from environment. Yet the control loop, thereupon released from the mechanical feedback, brings the 
system again into the contact. This, repeated over and over, hunting phenomenon [3] is well known in the practical force-controlled robotic applications, and has been recognized already in [13]. To overcome the hunting phenomenon, which can be seen as a local (to say transient) instability of the force control in vicinity to environmental contact, a velocity feedback loop is usually inserted. The system, adjusted in this way, can however become unstable for small velocity feedback gains, while the system response becomes remarkably slower for larger gain values, cf. [14].

Depending on the motion control task, e.g. position or force set-point (equally as trajectory tracking), a possibly large or respectively low stiffness has to be achieved via a dedicated control design. At the same time, the motion control problem can be specified in term of obtaining the desired mechanical impedance. That means controlling the motion dynamics to respond as a second-order system

$$
M_{d}\left(\ddot{X}_{d}-\ddot{X}\right)+D_{d}\left(\dot{X}_{d}-\dot{X}\right)+K_{d}\left(X_{d}-X\right)=F_{c},
$$

with the desired (apparent) mass $M_{d}$, desired damping $D_{d}$, and desired stiffness $K_{d}$. We recall that the latter is particularly characteristic for an impedance control which can be seen as trade-off between an absolutely "stiff" positioning and absolutely "soft" force following, cf. Figure 1. For the generalized dynamics (3) of an impedance-controlled motion system, the desired smooth motion reference is given by $X_{d}(t)$, while $F_{c}(t)$ is the contact force on interface with environment. One can recognize that for an ideal position control, with $X_{d}=0$ for simplicity and $K_{d} \rightarrow \infty$, an external contact force, as disturbance, should not cause any displacement, i.e. $X \rightarrow 0$. On the contrary, an ideal force control with $K_{d} \rightarrow 0$ will maintain the desired force while allowing, at the same time, for an unbounded displacement $X \rightarrow \infty$ of the apparent, to say virtual, mass with the shaped as desired damping. In other words, an impedance control is the general form of motion control [3], [5], since it is possible to turn the impedance control to both the position and force controllers, and that through a dedicated adjustment of control parameters. The concept of impedance control has been initially proposed by Hogan [15] and since then greatly recognized and elaborated, especially in robotic and control communities [11], [13], [16]. In particular for a safe and flexible human-machine interactions [17] and haptics [18], the impedance control and its various extensions have gained a remarkable recognition. With substantial progress in the sensing technologies and lightweight and soft in operation design of the robotic and mechatronic systems, it remains further on in focus of the active research, see for example in robotics the survey [19] and references therein. Since already in eighties it was recognized that both position and force cannot be controlled simultaneously, a hybrid position/force control was proposed in [20], based on an orthogonal decomposition of the task space. For multi-DOFs motion control, a full-dimensional task space is split into the so-called positioncontrolled and force-controlled subspaces. A dedicated selection (or switching) strategy endows the entire control system with a hybrid behavior, including variable structures and logic-based switching. One can also notice that with today's maturity of the hybrid systems theory [21], more and more capabilities arise for designing the dedicated hybrid position/force controllers, see e.g. [22], [23] for a recent example.

\section{Position and force controls require very high and, correspondingly, very low stiffness. Impedance control has finite stiffness in-between as application specific trade-off.}

\section{IDEAL VERSUS DISTURBED MOTION UNDER CONTROL}

The relative motion under control is usually achieved with some feed-forward and feedback regulators

- both designed, to the large part, for a nominal system plant. With physical Newton's laws, under 
Lagrange-d'Alembert principles, the modeled relative motion always assumes a forwarding of doubleintegrator between the generalized force and relative displacement caused by that. At the same time, for multi-body dynamics, arising from multiple constructive elements in a motion system, the chain of multiple integrators appears. This way forward propagation of the input force, correspondingly energy, results in an increasing system dynamics, see Figure 2. Note that the additional control and actuator elements, being located before the input force $F$, equally contain the coupled integrators, thus shaping the overall system dynamics. While all modeled forward and feedback couplings compose the particular system behavior, correspondingly determine the structure of the signals flow and associated differential equations, the motion disturbances can appear as both, exogenous quantities and/or functions of the system state, but in last case with unknown/uncertain relationships and parameters. Note that the structure depicted in Figure

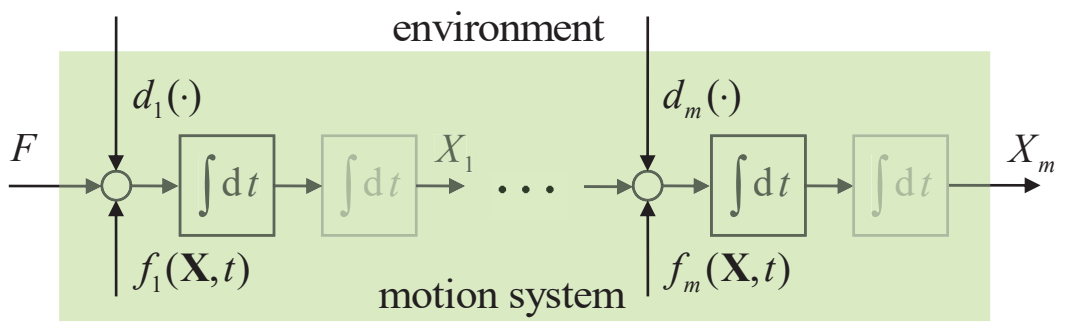

Fig. 2. Relative motion system with $m$ coupled double-integrators actuated by the generalized force $F$ and affected by nominal dynamics $f$ and unknown internal and external (environmental) disturbances $d$

2 represents a SISO (single-input-single-output) motion system, while it can equally capture the coupled SIMO (single-input-multiple-output) dynamics, provided the additional output channels are led out from the corresponding dynamic state variables. Example for such SIMO system is a rotational robotic joint with gear elasticities, see e.g. [16], [24], where both the motor and joint/link angular displacement appear as the output states of interest. Note that the motion systems with multiple input channels, i.e. MIMO (multiple-input-multiple-output), are omitted here for the sake of simplicity. Furthermore one should notice that, depending on some unconstrained and uncoupled motion variables, the free integrators can vanish or be neglected in the modeling, cf. grey-shadowed integrator blocks in Figure 2. Example for that is a linear damper (dashpot) element, within a chain of integrators, for which no inertial terms appear and the input force and output displacement are connected via the single integrator according to Newtonian's fluid. Another example is when a relative velocity of an unconstrained motion is the system output, thus resulting in elimination of one free integrator. Since the disturbance signals may come from environment, to say outside of the motion system, their origin and impact can be very different. Also in terms of the matching condition (see further in Sections VI.A and VI.B), the disturbances can be matched, like $d_{1}$ which is the most simple case, or mismatched as generalization i.e. $d_{m} \forall m \neq 1$. But as a common feature they mostly negatively affect the nominal system dynamics and, in consequence, impair performance of the motion control, or even destabilize it in the worst case. Apart from the mechanical and often most pronounced vibrational and friction disturbances (addressed further in Section III and IV respectively), other disturbing by-effects can appear in a closed-loop control system. Examples are the measured output bias or drift, addressed e.g. in [25], disturbing by-effects due to the control discretization [26], time-delay and communication disturbances in the loop [27], parameter and load disturbances [28], [29], and others.

Assuming the vectors of the system state $\mathbf{X}$, nominal system dynamics $f_{i}$, disturbances $d_{i}$, cf. Figure 2 
and, in addition, input force distribution $\mathbf{u}(\mathbf{X}, F)$, a generalized motion system can be approximated by

$$
\left(\begin{array}{c}
\dot{X}_{1} \\
\ddot{X}_{1} \\
\vdots \\
\dot{X}_{m} \\
\ddot{X}_{m}
\end{array}\right)=\left(\begin{array}{ccccc}
0 & 1 & \cdots & 0 & 0 \\
& & \frac{\partial}{\partial \mathbf{X}}\left(f_{1}+d_{1}\right) & & \\
& & \vdots & & \\
0 & 0 & \cdots & 0 & 1
\end{array}\right)\left(\begin{array}{c}
X_{1} \\
\dot{X}_{1} \\
\vdots \\
X_{m} \\
\dot{X}_{m}
\end{array}\right)+\left(\begin{array}{c}
0 \\
1 \\
\frac{\partial}{\partial \mathbf{x}} \mathbf{u}
\end{array}\right) F .
$$

Note that the vector $\mathbf{u}(\cdot)$ of the force distribution maps the input force $F$ to the lower-order motion variables $X_{i}$ and $\dot{X}_{i}$ with $i>1$, cf. Figure 2, thus capturing zero dynamics in the system. Following remarks can be made for the linearized modeling (4) of the motion system:

- If the linearized motion system (4) has no zeros, the force coupling vector results in $\partial \mathbf{u} / \partial \mathbf{X}=0$.

- The mismatched disturbances cannot be directly compensated by the control input force, cf. discussion provided in Section VI.B. The computed (model-based) compensation of mismatched disturbances will be inherently affected by the impact of (multiple) integrators in the chain.

- If disturbances are not the functions of the state and, hence, their partial derivative do not exist, then the corresponding terms should be removed from the linearized system matrix of (4) and collected as an additional vector, thus approaching (14) cf. Section V.B.

- The state-independent disturbances, which appear as exogenous values, cannot destabilize the eigenbehavior of system (4) but significantly affect its transient and steady-state response.

With respect to the said above, the most challenging issues for an advanced motion control are in the measurement or robust observation/estimation of various-type disturbances, and their localization and isolation as prerequisite for an efficient compensation. This is in addition to a sufficiently accurate modeling which, however, may be subject to both parametric and structural uncertainties, see e.g. [30].

\section{VibRATIONAL PERTURBATION IN STRUCTURES}

Vibration in structures, due to various kinds of mechanical resonances as well as electrical and/or physical linear/nonlinear properties, can deteriorate the motion control performances, especially for fastresponse and high-precision applications [1]. In order to effectively design the motion controller (on the levels of force, torque, velocity, and/or position) against the vibratory properties, in general, precise modeling and identification for the target systems should be indispensable for accurate model-based feedforward compensations and robust/adaptive feedback controllers. Perturbation in the vibratory properties, however, essentially inheres in the actual target systems and should strongly affect the higher motion control performances. In the mechatronic systems, the perturbation can appear as parameter/property variations in mechanical/electrical resonant frequency, damping/friction, actuator torque/force constants, etc. In this section, a key practical knowledge of vibrational perturbation due to structured/unstructured uncertainties [30] is surveyed, especially focusing on how this type perturbation occurs and how the effective modeling and controller should be designed.

\section{A. Changes in mechanical structure}

Changes and/or transformations in mechanical structures, such as, posture/load of industrial robots, work arrangements of machine tools, etc., directly vary equivalent moment of inertia as well as resonance frequency. Example is the linear trajectory motion for a 6-axis industrial robot, where the equivalent moment of inertia, especially in the first or second axis, is drastically changed according to the posture [31]. It leads to perturbed frequency Bode characteristics in the rigid mode gains as well as vibration mode characteristics (resonance/antiresonance frequencies and gains), resulting in deteriorating robot motion performances, e.g. accuracy in settling, trajectory, velocity, etc. In case of the first or second axis, since the inertia generally varies up to 3 to 4 times, it directly affects the control performance, such as bandwidth and stability of feedback system, and model-based feedforward compensation. In order to improve the 


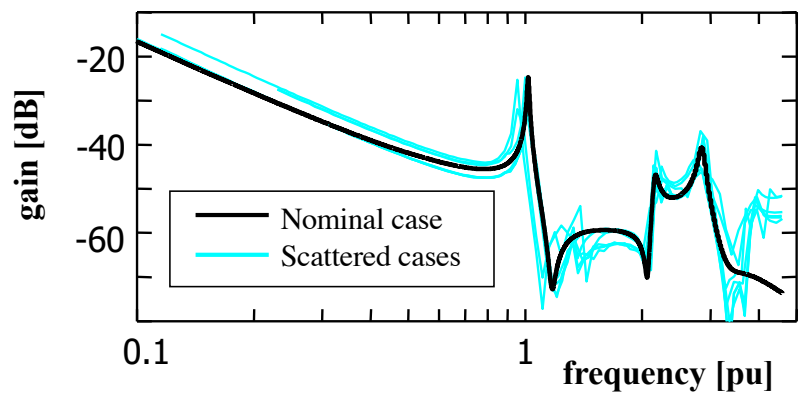

(a)

(b)

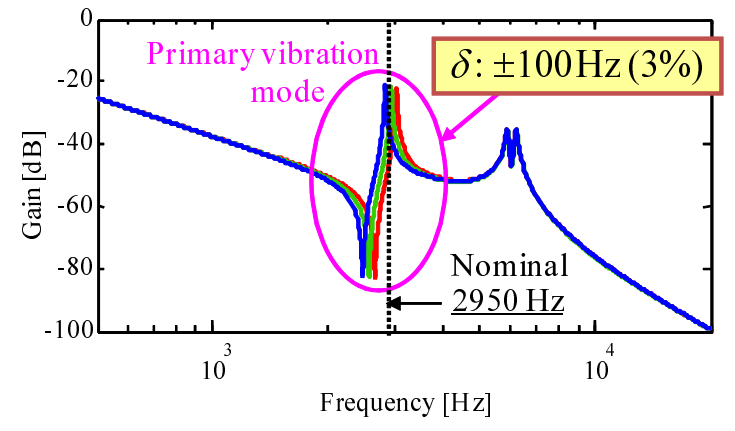

Fig. 3. (a) example of scattered Bode gain characteristics among mass productions of galvano scanners for laser-drilling machines. (b) gain diagrams of plant system with frequency variations $\delta= \pm 100 \mathrm{~Hz}$ in primary vibration mode due to temperature fluctuation during motion operations

motion control performance against the structural changes in mechanism, precise modeling/simulator based on kinematic analyses, variable structure control (e.g. variable filters [31]), adaptive algorithm, and robust control [32] should be promising to compensate for the variations. The CAD-supported kinematic analyses are also promising to specify the boundary of perturbations, following to the robust controller design.

\section{B. Scattered characteristics among mass productions}

In actual mass productions of industrial mechatronic systems, the dispersion of characteristics (in mechanical, electrical, and physical properties) essentially exists, which leads to the scattered motion performance, because a controller is usually designed using a typical characteristic for a series of products. Figure 3 (a) gives an example of scattered Bode gain diagrams among mass productions of galvano scanners for laser-drilling machines [1]. The scanner mechanism can be modeled by a multi-mass body system: a black line represents the gain characteristic in the nominal case, while blue lines represent the scattered ones among numerous products. From the figure, dispersion components are clearly observed in the rigid mode gain and frequency/gain of the primary, second, and third vibration modes, respectively. For this scanner positioning, the perturbation of some percentages leads to the drastic performance deteriorations with more than $10 \%$ error in the required specifications (settling time, accuracy, etc.); it is quite different situation comparing to the effects of perturbation due to changes in mechanical structure stated above.

In order to ensure the constant motion performance against the scattered characteristics, adaptive and/or robust control should be effective based on the precise modeling and practical estimation in the perturbation boundary, while online auto-tuning algorithm [33], [34] should be promising to perform the precise parameter selection in feedforward and feedback compensations, as well as to achieve the time and labor saving from the practical viewpoints in industrial applications.

\section{Changes in environmental conditions}

Also environmental conditions in mechatronic systems, such as temperature fluctuations, aged deteriorations (from some hours to years), system delay components (dead-time and servo lag in controller, and time delay in communication lines), unknown disturbances, etc., drastically affect the motion performances in a wide variety of actual motion operations. For example, the temperature fluctuations in mechanisms and/or electrical components can directly cause variations in the vibration modes (gain and frequency), damping and frictional forces, and actuator torque/force constant, due to the essential properties in mechanical/electrical materials (metals, magnets, etc.). Figure 3 (b) shows an example of perturbed gain characteristics of the galvano mirror, with frequency variations $\delta= \pm 100 \mathrm{~Hz}$ in the primary vibration mode due to temperature fluctuation during motion operations. As the result of perturbations, the settling 
(a)
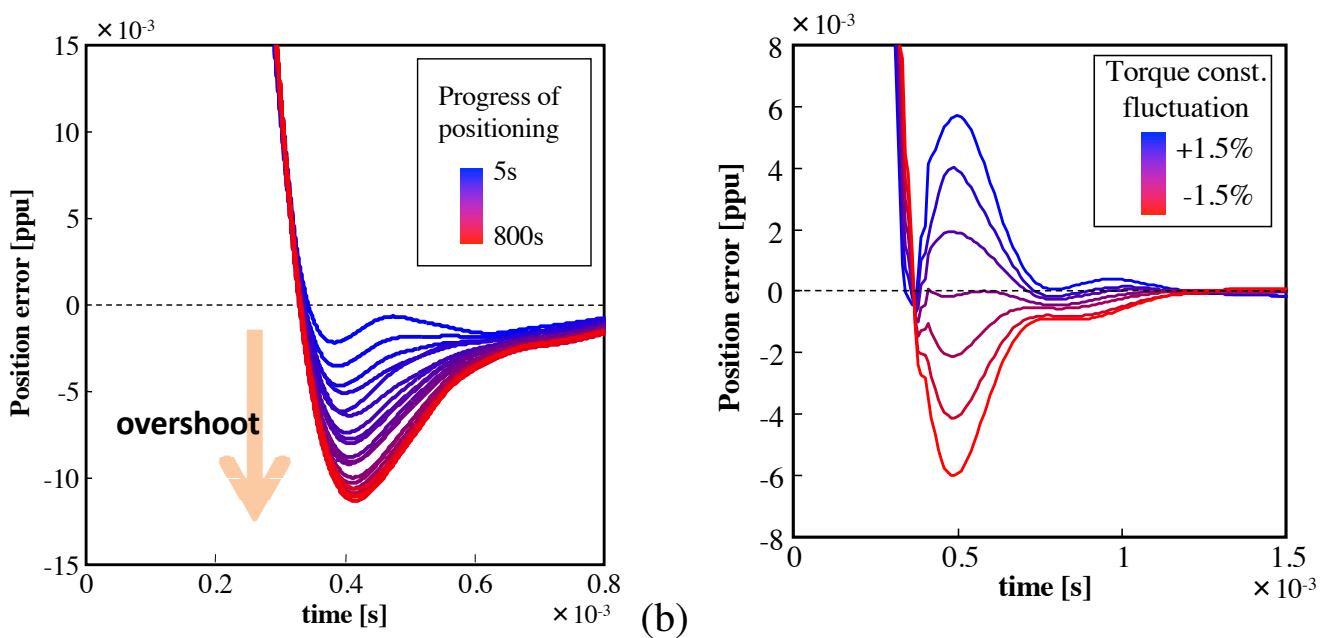

Fig. 4. (a) changes in response of position error due to vibrational perturbation as operations of galvano mirror positioning progress from $5 \mathrm{~s}$ to $800 \mathrm{~s}$ : temperature of mechanical/electrical components rises during the progress, leading to change in vibration mode and actuator torque constant. (b) deteriorations in settling performance due to fluctuation of actuator torque constant: overshoot and/or undershoot responses clearly occur corresponding to torque constant variation

performances in positioning are clearly deteriorated as shown in Figure 4 [35]. In the case of Figure 4 (a), as the positioning operations of galvano mirror progress from $5 \mathrm{~s}$ to $800 \mathrm{~s}$, the overshoot responses gradually increase due to the temperature rising in mechanism and actuator, which leads to the changes in vibration mode as well as the decrease in actuator torque constant. Figure 4 (b), on the other hand, clarifies that the fluctuation in actuator torque constant with the range of $\pm 1.5 \%$ deteriorates the position settling performances with overshoot and/or undershoot responses corresponding to the torque constant variation. In order to compensate for the environmental changes, the online auto-tuning and/or adaptive control are also promising, especially for the aged deteriorations in mechanisms and electrical components. Those algorithms, in addition, can apply to the cases caused by the machine setting environment with different floor conditions, leading to change in resonance frequency of machine stand vibration. As recent globalized manufacturing chains are rapidly expanding, these kinds of auto-tuning/adaptation algorithms should be powerful enough to eliminate the various environmental perturbations [36].

In conclusion of this section, the promising approaches and/or techniques, i.e., practical precise modeling and controller design, robust and/or adaptive control, and optimization (including online auto-tuning), should be applied to compensate for motion performance deteriorations due to the actual vibrational perturbation. In addition, those techniques may proceed to diagnosis and/or fault-tolerance algorithms to prevent unexpected accidents [37].

\section{FRICTIONAL PERTURBATION ON INTERFACES}

The motion control systems are usually equipped with various-type contact interfaces, due to bearing of the parts which move relative to each other. Exceptions, also encountered in the mechatronic design, are the air and magnetic bearings, see e.g. [38] and [39] for an example and overview respectively. Those both technologies, even though relevant and fairly challenging for design and motion control, are not directly associated with contact interfaces and related frictional perturbations. Because of that, they remain next out of our main focus. Once a pair of mechanical parts is in loaded physical contact, the corresponding (generalized) friction force appears on the interface and acts in direction opposite to the ongoing relative motion. Here we recall the statement that the friction is present in all machines incorporating parts with relative motion [40]. And although friction may be a desirable property, as it is for brakes, it is generally an impediment for servo control. Not surprisingly, the volume of research works dedicated to modeling, identification and, in the first line, compensation of the friction effects is huge and cannot be satisfactorily 


\section{Scattered characteristics in mass production, operational transformations of mechanical structure and environmental conditions of mechatronic systems considerably change their gain and modal properties. These changes significantly deteriorate the nominal motion control performance, especially in fast and high-precision applications.}

accommodated in this correspondence. At the same time, for a former and well-celebrated survey it is worth to refer to [40]. Also a more recent seminal work [41], dedicated to friction force dynamics, summarizes and highlights (ditto with references therein) the most pronounced phenomena associated with kinetic friction. Among those are the so-called presliding friction behavior, adhesion and creeping, transient frictional lag, well-known stick-slip motion, and others.

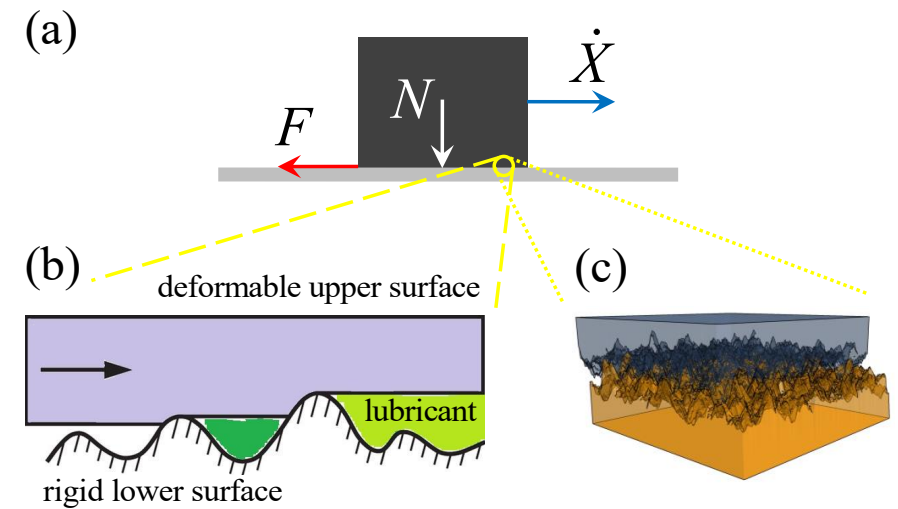

Fig. 5. Frictional appearance of contact interface. (a) contact pair of moving body on frictional surface with normal load $(N)$. (b) schematic representation of deformable upper surface moving along the rigid lower surface with lubricant penetration between asperities. (c) typical topology of the rough surface pair in contact.

The appearance of frictional forces on a contact interface, and that independently of the type of contact mechanisms like slider, ball-bearing, roller etc, can be imagined by inspecting principal configuration depicted in Figure 5 (a). A normal loaded (by the force $N$ ) contact pair induces the kinetic friction force $F$ counteracting relative displacement with the velocity $\dot{X}$. Sliding along each other, the outer surface layers deform elasto-plastically (for the sake of simplicity only one surface is drawn as deformable in Figure 5 (b)), while the surface roughness provides irregular contact asperities with lubricant medium distributed in between. Note that the squeezed lubricated regions, marked by the dark green in Figure 5 (b), are characterized by a hydrodynamic pressure of lubrication medium, that gives rise to an additional lift-up by-effect, cf. [41]. Despite irregular landscape of the rough surfaces in contact, cf. Figure 5 (c), a particular surface topology can be characterized by some average values of the asperities height, distance, distribution, and elasto-plastic characteristics.

Provided the existence of an accurate and real-time capable model of the friction forces, those can be compensated (at least theoretically) either in a feed-forward or feedback manner. This has been widely 
attempted in the formerly works since the kinetic friction, in general consensus, constitutes a deterministic nonlinear map between the arising friction force and relative displacement rate as its cause, i.e. $f: \dot{X}(t) \rightarrow$ $F(t)$. Despite a notable progress in the understanding and modeling of friction phenomena, the direct model-based compensation techniques fail, however, in most cases when it comes to a robust and practice suitable motion control, and that due to several reasons of quite different nature. The most apparent one is non-feasibility of a direct friction measurement on the contact interfaces. Since the friction force appears rather as a mismatched and internally coupled disturbance (cf. Figure 2 and related discussion on ideal versus disturbed motion), its proper decomposition and isolation within the total balance of forces almost always rely on some strong and often artificial assumptions, these without sensoric examination of the physical values. Therefore, the frictional quantities in servo-controlled machines are usually obtained as the residual values, out of the total dynamics balance, provided the other force components are properly identified and known based on the measurements. The next principal obstacle in dealing with accurate friction properties, lies in the (predominantly) spatial nature of the friction, while the residual terms of the system and control dynamics are specified and handled in time or frequency domain. Example for this misalignment in dynamics properties is a degraded integral control performance when attempting to compensate for presliding friction, cf. e.g. [42]. This issue has been analyzed and experimentally demonstrated in [9], see example shown in Figure 6. Here a well-tuned integral control part fails to ensure a fast settling behavior and results in a slow creeping of the controlled position response due to nonlinear presliding friction. Finally, the kinetic friction in the motion control systems is subject to large

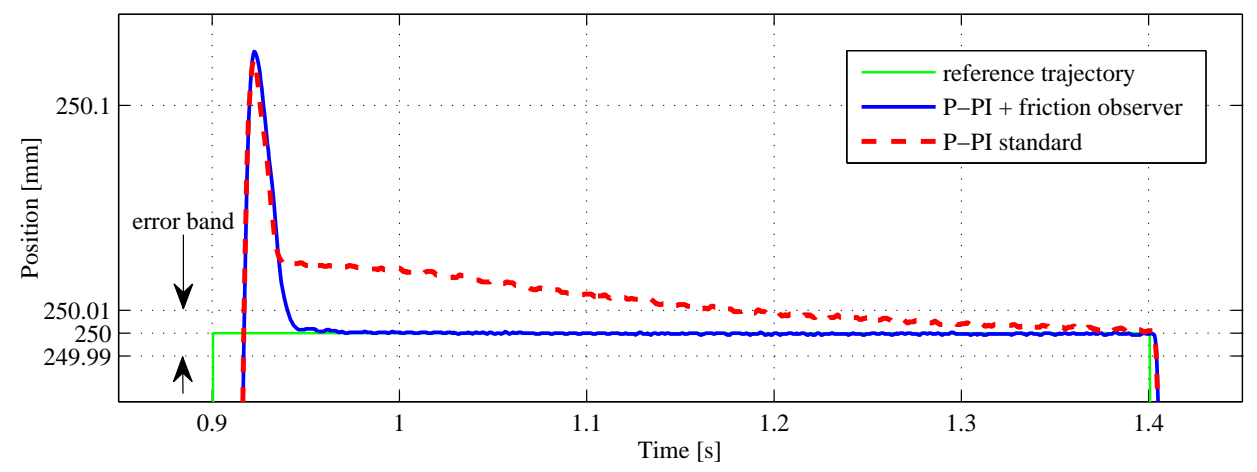

Fig. 6. Position response of the standard P-PI cascaded controller and that extended by an explicit friction observer (exemplified experimental evaluation on the ball-screw positioning stage from [9])

uncertainties due to the multiple, and often weakly known, internal and external factors such as roughness, thermal and lubricant state of contacting surfaces, varying normal loads, dwell time, wear, and others [43]. This fact requires an additional and above all fast estimation, correspondingly observation, of the transient and time-varying frictional states and/or parameters. Note that the latter can be often assumed as available, with the previously identified, to say nominal, values. Therefore, the classical adaptive (in parameters) strategies and algorithms render of less priority here, even though remaining still interesting for the control tuning. This is in case of the changeable loads, thermal and lubrication conditions, or control initialization when set into operation. The necessity of observing the friction disturbances has been already shown in [44] and remains further on in scope of the up-to-date research as has been demonstrated, for instance, for the absolute (encoder resolution) accuracy of positioning [43] and friction torque estimation in the robotic joints [24], [45].

While a generalized empirical friction model structure, according to [41], requires the friction force to be a (nonlinear) map $F=\mathcal{F}(X, \dot{X}, z)$ of equally the relative displacement and some internal state vector, which dynamics is driven by $\dot{z}=\mathcal{G}(X, \dot{X}, z)$, another general friction force mappings can be postulated as well. For the relative displacement rate, assumed as the main factor driving dynamics of the kinetic friction, one can write

$$
F=f(\dot{X}, t),
$$


where an explicit time argument captures the time-varying behavior and dynamic response of the friction force. In the most simple case of a static and, at the same time, most widespread description of the friction force, the relationship (5) transforms into $F=\alpha \dot{X}+\beta \operatorname{sign}(\dot{X})$, with $\alpha$ and $\beta$ to be the linear viscous and correspondingly Coulomb friction coefficients. In a more general dynamic case of the kinetic friction, a differential equation

$$
\dot{F}=g(F, \dot{X}, t),
$$

can be assumed instead of (5). Note that (6) is compliant with the Dahl friction model [46], in its original differential form. Recall that the Dahl model can be credited as a first but still relevant approach of capturing the transient presliding friction characteristics. If one assumes the total kinetic friction as some (general) superposition of the Coulomb- and viscous-type frictional terms, the eqs. (5) and (6) can be concretized as for example provided in [43]. When doing it, the frictional uncertainties will end up either in the lumped but time-varying friction parameters or unknown disturbance to be observed, as exemplified below.

\section{Frictional perturbations occurring on mechanical interfaces of a motion control system are state- and time-variant. The dynamics of kinetic friction is driven by the motion variables and prehistory of the friction force, while the parameters change by the thermal, roughness, lubrication, load and wear conditions of the contact surfaces.}

While disturbances due to unknown friction can be classified, more or less, by certain amplitude and frequency range or assumed as correspondingly steady-state upper bounded, the disturbances due to uncertain friction are often highly dynamical and transient in occurrence. It makes them particularly challenging for an appropriate estimation, as a convergence phase is required before an estimated frictional state can be used instead of a real physical value. This is generally independent of type of the designed observer. For instance, following the modeling approach [43] the dynamics (6) of friction force results in $g: \alpha \tau^{-1} \dot{X}-\tau^{-1} F+\beta \mathrm{d} / \mathrm{d} t \operatorname{sign}(\dot{X})$, where $\tau$ is the time constant of frictional lag. One can recognize that otherwise time-varying $\alpha(t), \beta(t)$ and $\tau(t)$ parameters, capturing uncertainties of frictional behavior, would significantly affect the friction force dynamics, and that in a coupled way challenging for a proper decomposition. It is also evident that the two times Dirac delta function, due to the sign discontinuity of the modeled Coulomb friction, is additionally weighted by, this case, varying $\beta(t)$ parameter. That leads to an uncertain excitation of the force dynamics every time the sign of the relative velocity changes. We also recall that in order to deal with force discontinuity at motion reversals, and due to the fact that relative motion never starts or stops abruptly but within certain (presliding) region of the relative displacement and, correspondingly, growing friction force, different approaches have been proposed. It has started by the Dahl model [46] and, since there, flourished in the number of approaches with different modeling complexity and level of details when capturing peresliding transitions. Along with several formulations based on nonlinear differential equations, the Maxwell-slip type structures, equivalent to the distributed Prandtl-Ishlinskii hysteresis operator [47], turned out to be particularly suitable for shaping hysteresis in the presliding force transitions. Further extensions of the principal Coulomb and viscous (including 
Stribeck effect) friction laws attempt capturing the stochastic aspects of rough surfaces, temporal and load-dependent relaxation, adhesion (also known as stiction), impact of the dwell time and "warming up" affects and others. At the same time, it is worth noting that, despite more elaborated friction models can yield a better consonance with experimental data, it is rather generality in the modeling of kinetic friction which remains, and perhaps will remain, one of the most appealing issues. Ensuring a sufficient trade-off between generality and level of details when describing the multifaceted phenomenon of kinetic friction should allow for an appropriate estimation and compensation of frictional perturbations in the controlled motion systems.

\section{MEASUREMENT AND OBSERVATION TECHNIQUES IN MOTION SYSTEMS}

Measurement and estimation techniques are becoming more important in motion systems. They not only provide information required by control systems but also facilitate many other important tasks including operating status monitoring, fault detection and diagnosis, and detecting the change of environment conditions. Sensors and physical devices are used to measure the state and workload of a dynamic system or sense the working/operational environment. With the advances of sensing technologies, more and more physical quantities are now available for measurement with an increasing accuracy. However, there is also a concern about cost, which is important for industrial mechatronic systems particularly these in mass production. Significant effort is also made in extracting or inferring as much as possible information from available measurements. A large number of estimation and observation techniques have been developed. Examples are the development of sensorless control in motion systems [48] where some key feedback measurements are replaced by estimation techniques. More recently developed is the so-called "virtual torsion sensor" [24] which allows reducing an output encoder in the robotic joints with non-negligible elasticities. They could be considered as soft sensor technologies, and quite often rely on a good model or knowledge of a motion system and its operational environment of concern. In this regard, based on their purposes, there are different types of observation techniques, namely state observation, disturbance observation, and joint state and disturbance estimation. It is worth of pointing out that in many applications, disturbance estimation techniques are not just used to estimate external disturbances but also the influence of system uncertainty in modeling, e.g. due to the mismatch between a model and physical system or the change of the motion system as a consequence of the change of operational conditions and environments, including system faults. In the following, we will discuss a number of existing and new observation technologies with focus on disturbance estimation as there exists a significant amount of literature on state observation, see e.g. [49] for overview.

\section{Robust estimation and observation algorithms serve as soft (virtual) sensors of unavailable dynamic states at almost zero hardware-costs.}

\section{A. Linear estimation techniques}

A wide range of observation technologies are available for motion systems described by linear dynamics. Classic Luenberger state observer [50] design technique for deterministic systems and Kalman filtering [51] for stochastic systems are well known for estimating unavailable system states from output measurements. This problem becomes slightly more complicated in the presence of external disturbances. A number of approaches have been developed for directly estimating the disturbance or jointly estimating the state and disturbance by augmenting the disturbance into the state so a conventional Luenberger state observer or Kalman filtering technique could be used. Depending on the description of the model of a motion system, transfer function and state space approaches have been developed. 
(a)

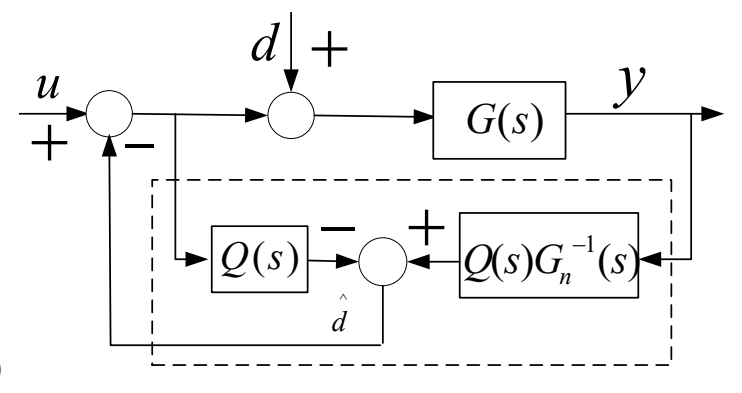

(b)

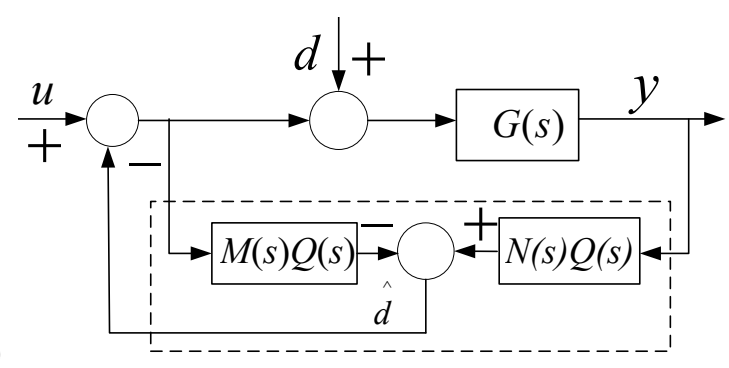

Fig. 7. (a) diagram of classic $Q$-filter based DOB as in [52]. (b) diagram of a generic DOB structure

1) Transfer functions approaches: The frequency-domain disturbance observer (DOB) was originally proposed in [52]. Suppose that a motion system is described by a transfer function $G(s)$. The basic idea in [52] is to obtain disturbance estimate by filtering the differences between control input and the calculated input using the inverse model of the nominal plant $G_{n}(s)$ through a low pass filter $Q(s)$. The basic diagram of DOB is given in Figure 7 (a), where $Q(s)$ is designed as a low-pass filter with unity gain. The relative degree of $Q(s)$, i.e. the order difference between the numerator and denominator, is not less than that of the nominal plant $G_{n}(s)$ to ensure that $Q(s) G_{n}^{-1}(s)$ is implementable. One promising feature of the disturbance observer techniques is that it is able to not only estimate external disturbance but also the influence of uncertainty, the so-called "lumped" disturbance concept. This could be illustrated by the diagram in Figure 7(a). Following block diagram manipulation, it can be shown that

$$
\hat{d}=Q(s)\left(\left(G_{n}^{-1}(s)-G^{-1}(s)\right) y+d\right)
$$

This implies that the disturbance estimate consists of two parts: the external disturbance $d$ and the mismatching between the physical system and its nominal model. Therefore, the disturbance observer techniques are able to estimate the influence of uncertainty. This is why they are also widely used as a robust control method as discussed in Section VI.

However, the original structure in [52] can not handle the non-minimum phase systems since the direct inverse of the nominal plant $G_{n}(s)$ brings unstable poles in $Q(s) G_{n}^{-1}(s)$. To this end, an improved and more generic version of DOB is given in [53]. It can be depicted as in Figure 7 (b), where $M(s)$ and $N(s)$ take the following form

$$
M(s)=\frac{M_{n}(s)}{L_{1}(s)}, N(s)=\frac{N_{n}(s)}{L_{1}(s)} .
$$

The nominal plant is represented as $G_{n}(s)=M_{n}(s) / N_{n}(s)$ with $L_{1}(s)$ being a stable polynomial, and $Q(s)$ is designed as a low-pass filter such that $M(s) Q(s)$ is a low-pass filter with unity gain. The key design parameter in this approach is the low-pass filter $Q(s)$, which depends on the frequency of the disturbance and uncertain dynamics to be estimated, the frequency of the sensor noise and the system dynamics. Guidance for how to design the disturbance observers through this approach can be found e.g. in [54].

2) State-space approaches: Shortly after the state observer technique (e.g. Luenberger observer [55]) was developed in 1960s, Johnson [56], [57] extended it to estimate external disturbance. This was realized by augmenting the system dynamics with a disturbance model so that the augmented system consists of both the system states and the disturbance (or the internal states of a disturbance dynamics). Therefore the system states and disturbance can be simultaneously estimated by using an existing state observation technique. Consider a single-input-single-output (SISO) linear system subjected to unknown disturbances, given by

$$
\left\{\begin{array}{l}
\dot{x}=A x+B_{u} u+B_{d} d \\
y=C x
\end{array}\right.
$$


where $x \in R^{n}, u \in R, d \in R$ and $y \in R$ are the system states, control input, disturbance and measurement, respectively. $A, B_{u}, B_{d}, C, D$ are the corresponding system matrices, which can be considered as a state-space realization of the nominal plant $G_{n}(s)$ in Figure 7 (a). The disturbance is supposed to be approximately represented by the following linear exogenous system

$$
\dot{\omega}=S \omega, \quad d=H \omega,
$$

where $\omega \in R^{q}$ and the pair $(S, H)$ is known and observable. It can represent a wide range of disturbances including unknown constant, ramp, and periodic signals such as sinusoid waves.

Combing the system dynamics (8) and disturbance dynamics (9), a composite system can be obtained:

$$
\left\{\begin{array}{l}
\dot{\bar{x}}=\bar{A} \bar{x}+\bar{B} u \\
y=\bar{C} \bar{x}
\end{array}\right.
$$

where $\bar{x}=\left[x^{T}, \omega^{T}\right]^{T}$, and the system matrices are given by

$$
\bar{A}=\left[\begin{array}{cc}
A & B_{d} H \\
O_{q \times n} & S
\end{array}\right], \bar{B}=\left[\begin{array}{c}
B_{u} \\
O_{q \times 1}
\end{array}\right], \bar{C}=\left[\begin{array}{ll}
C & O_{1 \times q}
\end{array}\right] .
$$

Following a standard state observer design procedure, the disturbance can be estimated by

$$
\left\{\begin{array}{l}
\dot{\bar{x}}=\bar{A} \hat{\bar{x}}+\bar{B} u+K(y-\bar{C} \hat{\bar{x}}), \\
\hat{\omega}=\bar{C} \hat{\bar{x}}, \\
\hat{d}=H \hat{\omega},
\end{array}\right.
$$

where $K$ is the observer gain matrix and hatted variables are the estimate of the corresponding variables. It is easy to see this approach could be extended to handle multiple disturbances and multiple-inputmultiple-output (MIMO) systems. For stochastic systems, Kalman filtering could be directly applied in a similar way.

The above approach is basically joint state and disturbance estimation. In some applications, only the disturbance is of interest, for example, where all the state are already available, or only faults or environment changes (which can be modeled as a type of disturbance) are of concern, a disturbance estimation could be developed using the so-called functional observer concept in the state-space approach [50]. This also provides a counterpart of the DOB design in the transfer function approach as described in Section V-A1.

A state-space disturbance observer is proposed in [58] using the functional observer concept as follows

$$
\left\{\begin{array}{l}
\dot{z}=F z+G y+T u, \\
\hat{\xi}=z+J y, \\
\hat{\omega}=\tilde{C} \hat{\xi} \\
\hat{d}=H \hat{\omega}
\end{array}\right.
$$

where $z$ is the state variables of the observer and $F, G, T$ and $J$ are the gain matrices designed to satisfy

$$
\left\{\begin{array}{l}
W \bar{A}=F W+G \bar{C}, \text { (Sylvester equation) } \\
W=L-J \bar{C}, \\
T=W \bar{B} \\
F \text { is stable, }
\end{array}\right.
$$

where $W$ is the intermediate matrix and $L$ is chosen based on the order of the observer to be designed. Under condition (13), one can prove that the disturbance estimation error converges to zero. It shall be noted in the above formula, the order of the disturbance observer could be changed by using the matrices with different dimensions, which is similar to choosing a different order of the $\mathrm{Q}$ filter in the transfer function approach. The design procedure can be found in [58]. The main difference between the functional disturbance observer in (12)-(13) and the disturbance observer in (10)-(11) is that it is much more flexible in choosing the order of the disturbance observer. Essentially, the disturbance observer (10)-(11) is a full order observer while the disturbance observer in (12)-(13) is a reduced order observer where the order could be determined by a designer (subject to the existence condition in [58]). 
Although there are similarities, the transfer function approaches and the state-space approaches have different features. For the further discussion of the relationships of the disturbance estimation between the transfer function and state-space approaches, we refer to [59] and [58].

\section{B. Nonlinear estimation techniques}

Estimating states and/or disturbances for a motion system described by nonlinear dynamics is much more challenging. In general, different from linear systems, there are no design methods of a nonlinear state observer for a general nonlinear system, although research has been conducted in developing nonlinear state observers for motion systems with a specific structure, e.g. [60]. Consequently it is even more difficult to design a joint state and disturbance observation for a nonlinear system. Therefore, the approach of augmenting the states with disturbances widely used in linear systems cannot be extended to nonlinear systems. However, there do exist several generic nonlinear disturbance observer techniques for nonlinear systems; for general form of nonlinear observer as system of singular first-order linear PDEs we also refer to [61].

In the following, consider a nonlinear system, in companion form, described by

$$
\left\{\begin{array}{l}
\dot{x}(t)=f(x(t))+g_{1}(x(t)) u+g_{2}(x(t)) d(t), \\
y(t)=h(x(t)) .
\end{array}\right.
$$

It is assumed that $f(x), g_{1}(x), g_{2}(x)$ are smooth functions in terms of the state $x$.

A number of techniques have been proposed to design nonlinear disturbance observers to estimate the disturbance $d$, see e.g. [62] and [49] for overview. A widely used one, sometimes also called 'Chen's disturbance observer' in order to distinguish from other nonlinear disturbance observer techniques, was proposed and evolved from an initial nonlinear disturbance observer presented in [63] for dealing with friction in a robotic manipulator system. Next we will introduce this nonlinear disturbance observer technique as below:

$$
\left\{\begin{array}{l}
\dot{z}=-l(x) g_{2}(x) z-l(x)\left[g_{2}(x) p(x)+f(x)+g_{1}(x) u\right] \\
\hat{d}=z+p(x)
\end{array}\right.
$$

where $z \in R$ is the internal state variables of the observer and $p(x) \in R$ is an auxiliary variable to be designed. The nonlinear observer gain $l(x)$ is then determined by

$$
l(x)=\frac{\partial p(x)}{\partial x} .
$$

The nonlinear disturbance observer asymptotically estimates the disturbance if the observer gain $l(x)$ is chosen such that

$$
\dot{e}_{d}=-l(x) g_{2}(x) e_{d}
$$

is asymptotically stable regardless of $x$, where $e_{d}=d-\hat{d}$ is the disturbance estimation error.

There are many ways to choose the nonlinear gain $l(x)$ and $p(x)$ such that (17) is stable and (16) is satisfied. A systematic approach was suggested in [64]. For a nonlinear system with a well defined relative degree $\rho$ from the disturbance to the output (i.e. the order difference between the highest derivative of the disturbance and the output), $p(x)$ is suggested as

$$
p(x)=p_{0} L_{f}^{\rho-1} h(x),
$$

where $L_{f}^{\rho-1} h(x)$ denotes the Lie derivative defined as the $(\rho-1)$ th derivative of the function $h(x)$ along the function $f$, see e.g. [65] for basics. Consequently, the nonlinear observer gain $l(x)$ is given by

$$
l(x)=p_{0} \frac{\partial L_{f}^{\rho-1} h(x)}{\partial x} .
$$


Therefore, the whole design process boils down to the problem of choosing the parameter $p_{o}$, which is a tuning parameter for the compromise between the convergence rate and the sensitivity to noise. Once this parameter is chosen, the nonlinear observer gain $l(x)$ and the auxiliary variable $p(x)$ are determined by (19) and (18), respectively. Then the nonlinear disturbance observer is given by (15). This nonlinear disturbance observer technique has been widely applied in motion and other mechatronic systems.

In addition to its simplicity in design, a most promising feature of the above disturbance observer design method is that the controller design could be separated from the disturbance observer design. Note that this somehow extends the so-called separation principle of state observer-based control design for linear systems. The separation principle, more formally known as the principle of separation of estimation and control, implies that the state observer design and controller design could be separated from each other. First, a state feedback control law is designed under the assumption that all the state are available. A state observer is then designed to estimate the state. Finally the feedback states are replaced by their estimates yielded by the state observer. In a similar fashion, a feedforward compensation could be designed under the assumption that the disturbance is measured (see the next section for the design methods), and then it is replaced by its estimate yielded by the nonlinear disturbance observer given in (15). Therefore, the control design and the disturbance observer design can be separated for disturbance attenuation and robustness of nonlinear systems.

The above separation for nonlinear systems [66], [67] is realized due to the special feature of the proposed nonlinear disturbance observer (15). That is, the convergence of the disturbance estimation is achieved irrespective of the state of the nonlinear systems. Consequently, the Chen's nonlinear disturbance observer can be combined with arbitrary nonlinear control design method, to improve its disturbance rejection or/and robustness under certain conditions. This feature makes the nonlinear disturbance observer and its control design framework (i.e. DOBC) attractive, thus becoming a successful design method in the area.

\section{CONTROL AND COMPENSATION TECHNIQUES IN MOTION CONTROL}

In addition to tradition PID controllers or alike, a wide range of advanced control methods have been developed and applied on motion control systems, including model predictive control, robust control, adaptive control, slide model control, internal mode control, output regulation, passivity based control, and active disturbance rejection control (ADRC). The amount of related control literature is too huge to be properly listed here, while for some survey and overview sources we refer to e.g. [1], [65], [68]-[72]

In this section, we will exemplarily focus on a new type of composite control method. This control mechanism, with two-degrees-of-freedom control structure, tries to exploit of both feedback and feedforward strategies and combine them together to achieve high performance in tracking/regulaiton, good stability and disturbance rejection, and strong robustness. More specifically, a feedback and feed-forward control strategy has been extensively discussed in [1] where a feed-forward controller that directly generates feed-forward control signal based on reference/command is integrated with a feedback controller that is driven by the control error. Another two-degrees-of-freedom robust control design has been shown for motion control of servomotor [28] based on coprime factorization by the Youla-Kucera parametrization technique, see e.g. [73] for details. Another widely used way to employ feed-forward is to attenuate the influence of disturbance by generating a compensation based on disturbance measurement. However, since the disturbance or the influence of uncertainties may not be available, correspondingly measurable, the feed-forward strategy is facilitated by the disturbance estimation techniques as introduced in Section V. DOBC is one of this type of composite control methods. Thanks to the separation principle as discussed above, a design procedure for developing a composite control scheme for motion systems is outlined below.

Step 1: Design a feedback controller to achieve stability and tracking/regulaiton performance without considering uncertainties;

Step 2: Develop a feed-forward control strategy to reject disturbance or uncertainties under the assumption that they are measurable; 
Step 3: Design a disturbance observer to estimate disturbance or the influence of uncertainties;

Step 4: Integrate the feedback and feed-forward strategy with the disturbance replaced by its estimate to constitute a composite controller and analyze its performance.

Design of a feedback controller in Step 1 could be carried out with any suitable design methods, e.g. PID, MPC, LQR, while several techniques of disturbance observer design in Step 3 have been introduced in Section V, depending whether the dynamics system is liner or nonlinear. In what follows, we will discuss how to design the feed-forward compensation to reduce the influence of disturbance and uncertainties in Step 2. There are a wide range of disturbances and uncertainties in motion control systems. For an example of AC motor driving systems these are the mechanical parameters such as the changes of the inertia, electrical parameters such as the changes of stator resistance due to temperature; friction torque; load torque; cogging torque; flux harmonic torque; distortion voltage; current offset errors; skewed slot torque, etc. Obviously it is important to improve the disturbance rejection and robustness of the controlled motor drive system.

\section{A. Matched Disturbance and Uncertainty Attenuation}

Disturbance and (or) uncertainty satisfying the matching condition implies that the disturbance or the effect of the uncertainty are applied in the same channel as the control input, or more precisely, the influence of a disturbance or uncertainty can be equivalent to the input channels in some way. Almost all the current transfer function based composite design approach (e.g. DOB) has this assumption explicitly or implicitly. For a system described by a state-space model as (8), the matching condition is met when $B_{d}=B_{u}$ (or, more precisely, $B_{d}=B_{u} \Gamma$ for some $\Gamma$ ). Similarly, the matching condition is satisfied if $g_{1}(x)=g_{2}(x)$ in (14) for nonlinear systems.

As the control input and the disturbance are in the same channel, the feed-forward compensation strategy is quite straightforward. That is, the influence of the disturbance could be completely removed (at least theoretically) by generating a counteract control action. Consider a composite controller is given by

$$
u=u_{f b}+u_{f f},
$$

where $u_{f b}$ is control action calculated by a feedback controller designed in Step 1 while $u_{f f}$ denotes the feed forward control. Consequently, after replacing the true disturbance with its estimate, the corresponding composite control law in the presence of matched disturbance is given by

$$
u=u_{f b}+u_{f f}=u_{f f}-\hat{d},
$$

where $\hat{d}$ is the estimate of the lumped disturbance. It can be shown that the influence of the disturbance or uncertainty could be completely removed if the estimate yielded by a disturbance observer approaches the real disturbances [64].

\section{B. Mismatched Disturbance and Uncertainty Attenuation}

Quite often the disturbance and control do not occur in the same channel so do to satisfy the matching condition. It is, in general, true when considering the influence of uncertainties as a part of disturbances. Mismatched disturbance and uncertainty widely exist in practical applications. In a servo control system, the disturbance could be, for example, a load torque but the control is the voltage applied to the motor, so that they do not appear in the same channel. An electric driving system has to generate corresponding current in the motor (which is a state of the motor) in order to counteract the external load torque. This implies some states cannot approach to zero in steady-state in the presence of mismatched disturbances/uncertainties.

The key issue for compensation of the mismatched disturbance using feed-forward strategy is to find a compensation action based on the estimate for general dynamic systems. This issue has been answered not only for linear systems but also for nonlinear systems [74], [75] where a systematic approach to design 
disturbance compensation gain that is able to remove the influence of mismatched disturbance/uncertainty from the output, at least in steady-state, is proposed. For a linear system (8), the disturbance compensation gain is given by [74]

$$
K_{d}=-\left[C\left(A+B_{u} K_{x}\right)^{-1} B_{u}\right]^{-1} C\left(A+B_{u} K_{x}\right)^{-1} B_{d},
$$

where $K_{x}$ is the feedback control gain. It can be shown that the above compensation gain reduces to $K_{d}=-1$ in the case of matched disturbance as in (21). This is because $B_{d}=B_{u}$ in that case. Therefore, the matched case could be considered as a special case of the design for the mismatched case.

If all state variables are measurable, a reduced-order DOB (for example, the functional observer based design [58] discussed in Section V) can be used and the following composite control law is employed

$$
u=K_{x} x+K_{d} \hat{d} .
$$

In the case that the state variables are unmeasurable, a joint state and disturbance observer could be employed. The composite control law in this case is designed as

$$
u=K_{x} \hat{x}+K_{d} \hat{d}
$$

where $\hat{x}$ and $\hat{d}$ are yielded by state and disturbance observers.

Now we consider a single-input single-output nonlinear system (14). The disturbance can be removed from the output channel in steady-state by the following composite control law [75]

$$
u=\alpha(x)+\beta(x) v+\gamma(x) \hat{d},
$$

where $\hat{d}$ is the disturbance estimate obtained by the nonlinear disturbance observer,

$$
\alpha(x)=-\frac{L_{f}^{\sigma} h(x)}{L_{g} L_{f}^{\sigma-1} h(x)}, \beta(x)=\frac{1}{L_{g} L_{f}^{\sigma-1} h(x)},
$$

and $\sigma$ is the input to output relative degree [65]. The disturbance compensation gain is designed by

$$
\gamma(x)=-\frac{\sum_{i=1}^{\sigma-1} c_{i} L_{p} L_{f}^{i-1} h(x)+L_{p} L_{f}^{\sigma-1} h(x)}{L_{g} L_{f}^{\sigma-1} h(x)},
$$

and

$$
v=-\sum_{i=0}^{\sigma-1} c_{i} L_{f}^{i} h(x),
$$

where $c_{i}$ are the coefficients to be selected. Observing (22) reveals key challenges in developing a feedforward strategy to reject mismatched disturbances. Different from the matched disturbances, the compensation gain in generally depends on the feedback control strategy designed in Step 1 and the corresponding gain. This is also true for nonlinear cases and the above result has been extended to other control strategies such as slide mode control. However, more research is still required for developing feedforward strategy for other types of feedback control design.

\section{CONCLUSIONS}

In this communication the long known, equally as recent, issues in the motion control have been summarized and discussed in a generic manner. It is recalled that all types of the motion control like position, force, and impedance control rest on the same principles of the motion stiffness. For making the latter easy manageable for the application-specific tasks, a well elaborated motion control design should have an inner acceleration control structure. That one has to ensure that various-type disturbances and uncertainties are sufficiently compensated, yielding the residual motion dynamics ideally as a system with double-integrators. Despite considerable progress in more advanced and more and more economical sensor technologies, it is often the inaccessibility of several process variables and cost factors of the hardware 
that require the stable and robust observation and estimation techniques to be developed and deployed as soft (to say virtual) sensors. Our discussion in these regards has been tailored into two parts. First, we brought into light both main sources of the system internal motion disturbances - by structures with associated vibrations and by contact interfaces with associated friction. Then, some advanced observation techniques useful for motion systems have been explained together with the control and compensation strategies. As such, this overview may contribute to better encircle the research problems in the motion control, which itself opens new opportunities for advanced mechatronic products and applications.

\section{REFERENCES}

[1] M. Iwasaki, K. Seki, and Y. Maeda, "High-precision motion control techniques: A promising approach to improving motion performance," IEEE Industrial Electronics Magazine, vol. 6, no. 1, pp. 32-40, 2012.

[2] M. Steinbuch and M. L. Norg, "Advanced motion control: An industrial perspective," European Journal of Control, vol. 4, no. 4, pp. 278-293, 1998.

[3] K. Ohnishi, M. Shibata, and T. Murakami, "Motion control for advanced mechatronics," IEEE/ASME transactions on mechatronics, vol. 1 , no. 1 , pp. 56-67, 1996.

[4] H. S. Lee and M. Tomizuka, "Robust motion controller design for high-accuracy positioning systems," IEEE Transactions on Industrial Electronics, vol. 43, no. 1, pp. 48-55, 1996.

[5] O. Khatib, "A unified approach for motion and force control of robot manipulators: The operational space formulation," IEEE Journal on Robotics and Automation, vol. 3, no. 1, pp. 43-53, 1987.

[6] K. Ohnishi, N. Matsui, and Y. Hori, "Estimation, identification, and sensorless control in motion control system," Proc. IEEE, vol. 82, no. 8, pp. 1253-1265, 1994.

[7] B. A. Francis and W. M. Wonham, “The internal model principle of control theory," Automatica, vol. 12, no. 5, pp. 457-465, 1976.

[8] R. D. Lorenz, T. A. Lipo, and D. W. Novotny, "Motion control with induction motors," Proc. IEEE, vol. 82, no. 8, pp. 1215-1240, 1994.

[9] M. Ruderman and M. Iwasaki, "Analysis of linear feedback position control in presence of presliding friction," IEEJ Journal of Industry Applications, vol. 5, no. 2, pp. 61-68, 2016.

[10] J. Luh, M. Walker, and R. Paul, "Resolved-acceleration control of mechanical manipulators," IEEE Transactions on Automatic Control, vol. 25 , no. 3, pp. 468-474, 1980.

[11] R. J. Anderson and M. W. Spong, "Hybrid impedance control of robotic manipulators," IEEE Journal on Robotics and Automation, vol. 4, no. 5, pp. 549-556, 1988.

[12] W. K. Chung, L.-C. Fu, and T. Kröger, "Motion control," in Springer handbook of robotics, pp. 163-194, 2016.

[13] C. H. An and J. M. Hollerbach, "Dynamic stability issues in force control of manipulators," in American Control Conference (ACC'87), pp. 821-827, 1987.

[14] S. Katsura, Y. Matsumoto, and K. Ohnishi, "Analysis and experimental validation of force bandwidth for force control," IEEE Transactions on Industrial Electronics, vol. 53, no. 3, pp. 922-928, 2006.

[15] N. Hogan, "Impedance control: An approach to manipulation," Journal of dynamic systems, measurement, and control, vol. 107, no. 17, 1985.

[16] A. Albu-Schäffer, C. Ott, and G. Hirzinger, "A unified passivity-based control framework for position, torque and impedance control of flexible joint robots," The international journal of robotics research, vol. 26, no. 1, pp. 23-39, 2007.

[17] E. Magrini, F. Flacco, and A. De Luca, "Control of generalized contact motion and force in physical human-robot interaction," in IEEE international conference on robotics and automation (ICRA2015), pp. 2298-2304, 2015.

[18] K. Ohnishi, S. Katsura, and T. Shimono, "Motion control for real-world haptics," IEEE Industrial Electronics Magazine, vol. 4, no. 2, pp. 16-19, 2010.

[19] A. Ajoudani, A. M. Zanchettin, S. Ivaldi, A. Albu-Schäffer, K. Kosuge, and O. Khatib, "Progress and prospects of the human-robot collaboration," Autonomous Robots, vol. 42, no. 5, pp. 957-975, 2018.

[20] M. H. Raibert, J. J. Craig et al., "Hybrid position/force control of manipulators," Journal of Dynamic Systems, Measurement, and Control, vol. 103, no. 2, pp. 126-133, 1981.

[21] R. Goebel, R. G. Sanfelice, and A. R. Teel, "Hybrid dynamical systems," IEEE Control Systems Magazine, vol. 29, no. 2, pp. 28-93, 2009.

[22] P. Pasolli and M. Ruderman, "Hybrid state feedback position-force control of hydraulic cylinder," in 2019 IEEE International Conference on Mechatronics (ICM), vol. 1, pp. 54-59, 2019.

[23] M. Ruderman, "On switching between motion and force control," in IEEE 27th Mediterranean Conference on Control and Automation (MED’19), pp. 445-450, 2019.

[24] M. Ruderman and M. Iwasaki, "Sensorless torsion control of elastic-joint robots with hysteresis and friction," IEEE Transactions on Industrial Electronics, vol. 63, no. 3, pp. 1889-1899, 2015.

[25] R. Antonello, K. Ito, and R. Oboe, "Acceleration measurement drift rejection in motion control systems by augmented-state kinematic kalman filter," IEEE Transactions on Industrial Electronics, vol. 63, no. 3, pp. 1953-1961, 2015.

[26] H. Fujimoto, A. Kawamura, and M. Tomizuka, "Generalized digital redesign method for linear feedback system based on n-delay control," IEEE/ASME transactions on mechatronics, vol. 4, no. 2, pp. 101-109, 1999.

[27] K. Natori, R. Oboe, and K. Ohnishi, "Stability analysis and practical design procedure of time delayed control systems with communication disturbance observer," IEEE Transactions on Industrial Informatics, vol. 4, no. 3, pp. 185-197, 2008. 
[28] T. Umeno and Y. Hori, "Robust speed control of dc servomotors using modern two degrees-of-freedom controller design," IEEE Transactions on industrial electronics, vol. 38, no. 5, pp. 363-368, 1991.

[29] J. S. Bang, H. Shim, S. K. Park, and J. H. Seo, "Robust tracking and vibration suppression for a two-inertia system by combining backstepping approach with disturbance observer," IEEE Transactions on Industrial Electronics, vol. 57, no. 9, pp. 3197-3206, 2009.

[30] J. C. Doyle, B. A. Francis, and A. R. Tannenbaum, Feedback control theory. Courier Corporation, 2013.

[31] M. Iwasaki, "Modeling and compensation for angular transmission errors for precision positioning devices with harmonic drive gearings," in Proc. of 6th International Conference on Positioning Technology, pp. 13-20, 2014.

[32] M. Iwasaki, "Robust full-closed control-based vibration suppression for positioning devices with strain wave gearing," in 12th FranceJapan and 10th Europe-Asia Congress on Mechatronics, pp. 11-17, 2018.

[33] M. Iwasaki, Y. Nagai, M. Kawafuku, and H. Hirai, "GA-based practical on-line auto-tuning for fast and precise positioning controller," in Proc. Seventh International Conference on Motion and Vibration Control, vol. 49, pp. 1-6, 2004.

[34] M. Yamamoto, Y. Okitsu, and M. Iwasaki, "GA-based auto-tuning of vibration suppression controller for positioning devices with strain wave gearings," in 2015 IEEE International Conference on Mechatronics (ICM), pp. 614-619, 2015.

[35] D. Matsuka, T. Tanaka, and M. Iwasaki, "Thermal demagnetization compensation for fast and precise positioning in galvanometer scanners," IEEE Transactions on Industrial Electronics, vol. 63, no. 9, pp. 5514-5522, 2016.

[36] K. Itoh, M. Iwasaki, and N. Matsui, "GA-based autonomous design of robust fast and precise positioning considering machine stand vibration suppression," in Second International Conference on Power Electronics, Machines and Drives (PEMD 2004), vol. 2, pp. 709-714, 2004.

[37] D. Matsuka and M. Tokuyama, "Deterioration diagnosis method for ball bearings that continue minute swaying motion," Journal of Advanced Mechanical Design, Systems, and Manufacturing, vol. 7, no. 1, pp. 30-38, 2013.

[38] H. Zhu and D. B. Bogy, "Hard disc drive air bearing design: modified direct algorithm and its application to slider air bearing surface optimization," Tribology international, vol. 37, no. 2, pp. 193-201, 2004.

[39] H. Bleuler, M. Cole, P. Keogh, R. Larsonneur, E. Maslen, Y. Okada, G. Schweitzer, A. Traxler et al., Magnetic bearings: theory, design, and application to rotating machinery. Springer, 2009.

[40] B. Armstrong-Hélouvry, P. Dupont, and C. C. De Wit, "A survey of models, analysis tools and compensation methods for the control of machines with friction," Automatica, vol. 30, no. 7, pp. 1083-1138, 1994.

[41] F. Al-Bender and J. Swevers, "Characterization of friction force dynamics," IEEE Control Systems Magazine, vol. 28, no. 6, pp. 64-81, 2008.

[42] R. Beerens, A. Bisoffi, L. Zaccarian, W. Heemels, H. Nijmeijer, and N. van de Wouw, "Reset integral control for improved settling of pid-based motion systems with friction," Automatica, vol. 107, pp. 483-492, 2019.

[43] M. Ruderman and M. Iwasaki, "Observer of nonlinear friction dynamics for motion control," IEEE Transactions on Industrial Electronics, vol. 62, no. 9, pp. 5941-5949, 2015.

[44] M. Iwasaki, T. Shibata, and N. Matsui, "Disturbance-observer-based nonlinear friction compensation in table drive system," IEEE/ASME transactions on mechatronics, vol. 4, no. 1, pp. 3-8, 1999.

[45] M. J. Kim, F. Beck, C. Ott, and A. Albu-Schaeffer, "Model-free friction observers for flexible joint robots with torque measurements," arXiv preprint arXiv:1907.00553, 2019.

[46] P. R. Dahl, "Solid friction damping of mechanical vibrations," AIAA journal, vol. 14, no. 12, pp. 1675-1682, 1976.

[47] M. Ruderman and D. Rachinskii, "Use of Prandtl-Ishlinskii hysteresis operators for Coulomb friction modeling with presliding," in Journal of Physics: Conference Series, vol. 811, no. 1, p. 012013, 2017.

[48] J. P. Johnson, M. Ehsani, and Y. Guzelgunler, "Review of sensorless methods for brushless DC," in IEEE Industry Applications Conference, pp. 143-150, 1999.

[49] W.-H. Chen, J. Yang, L. Guo, and S. Li, "Disturbance-observer-based control and related methods - an overview," IEEE Transactions on Industrial Electronics, vol. 63, no. 2, pp. 1083-1095, 2015.

[50] D. G. Luenberger, "An introduction to observers," IEEE Transactions on Automatic Control, vol. 16, no. 6, pp. 596-602, 1971.

[51] R. E. Kalman, "A new approach to linear filtering and prediction problems," Journal of basic Engineering, vol. 82, no. 1, pp. 35-45, 1960.

[52] K. Ohishi, M. Nakao, K. Ohnishi, and K. Miyachi, "Microprocessor-controlled dc motor for load-insensitive position servo system," IEEE transactions on industrial electronics, no. 1, pp. 44-49, 1987.

[53] Y. Choi, K. Yang, W. K. Chung, H. R. Kim, and I. H. Suh, "On the robustness and performance of disturbance observers for second-order systems," IEEE Transactions on Automatic Control, vol. 48, no. 2, pp. 315-320, 2003.

[54] E. Sariyildiz and K. Ohnishi, "A guide to design disturbance observer," Journal of Dynamic Systems, Measurement, and Control, vol. 136, no. 2, p. 021011, 2014.

[55] D. G. Luenberger, "Observing the state of a linear system," IEEE Transactions on Military Electronics, vol. 8, no. 2, pp. 74-80, 1964.

[56] C. Johnson, "Optimal control of the linear regulator with constant disturbances," IEEE Transactions on Automatic Control, vol. 13, no. 4, pp. 416-421, 1968.

[57] C. Johnson, "Further study of the linear regulator with disturbances - the case of vector disturbances satisfying a linear differential equation," IEEE Transactions on Automatic Control, vol. 15, no. 2, pp. 222-228, 1970.

[58] J. Su, W.-H. Chen, and J. Yang, "On relationship between time-domain and frequency-domain disturbance observers and its applications," Journal of Dynamic Systems, Measurement, and Control, vol. 138, no. 9, 2016.

[59] E. Schrijver and J. Van Dijk, "Disturbance observers for rigid mechanical systems: equivalence, stability, and design," Journal of Dynamic Systems, Measurement, and Control, vol. 124, no. 4, pp. 539-548, 2002.

[60] T.-S. Low, T.-H. Lee, and K.-T. Chang, "A nonlinear speed observer for permanent-magnet synchronous motors," IEEE Transactions on Power Electronics, vol. 40, no. 3, pp. 307-316, 1993.

[61] N. Kazantzis and C. Kravaris, "Nonlinear observer design using Lyapunov's auxiliary theorem," Systems \& Control Letters, vol. 34, no. 5, pp. 241-247, 1998. 
[62] X. Chen, T. Fukuda, and K. D. Young, "A new nonlinear robust disturbance observer," Systems \& control letters, vol. 41, no. 3, pp. 189-199, 2000.

[63] W.-H. Chen, D. J. Ballance, P. J. Gawthrop, and J. O'Reilly, "A nonlinear disturbance observer for robotic manipulators," IEEE Transactions on industrial Electronics, vol. 47, no. 4, pp. 932-938, 2000.

[64] W.-H. Chen, "Disturbance observer based control for nonlinear systems," IEEE/ASME transactions on mechatronics, vol. 9, no. 4, pp. 706-710, 2004.

[65] A. Isidori, Nonlinear Control Systems: An Introduction, 3rd ed. Springer-Verlag, 1995.

[66] K. J. Astrom, Introduction to stochastic control theory. Academic Press, 1970.

[67] S. Li, J. Yang, W.-H. Chen, and X. Chen, Disturbance observer-based control: methods and applications. CRC Press, 2014.

[68] W. Perruquetti and J.-P. Barbot, Sliding mode control in engineering. CRC press, 2002.

[69] R. Findeisen, L. Imsland, F. Allgower, and B. A. Foss, "State and output feedback nonlinear model predictive control: An overview," European journal of control, vol. 9, no. 2-3, pp. 190-206, 2003.

[70] R. Ortega and E. Garcia-Canseco, "Interconnection and damping assignment passivity-based control: A survey," European Journal of control, vol. 10, no. 5, pp. 432-450, 2004.

[71] A. Sabanovic, "Variable structure systems with sliding modes in motion control - a survey," IEEE Transactions on Industrial Informatics, vol. 7, no. 2, pp. 212-223, 2011.

[72] T. Oomen, R. van Herpen, S. Quist, M. van de Wal, O. Bosgra, and M. Steinbuch, "Connecting system identification and robust control for next-generation motion control of a wafer stage," IEEE Transactions on Control Systems Technology, vol. 22, no. 1, pp. 102-118, 2013.

[73] M. Vidyasagar, Control System Synthesis: A Factorization Approach. Morgan \& Claypool Publishers, 2011.

[74] J. Yang, W. Chen, and S. Li, "Non-linear disturbance observer-based robust control for systems with mismatched disturbances/uncertainties," IET Control Theory and Application, vol. 5, no. 18, pp. 2053-2062, 2011.

[75] J. Yang, S. Li, and W. Chen, "Nonlinear disturbance observer-based control for multi-input multi-output nonlinear systems subject to mismatching condition," International Journal of Control, vol. 8, no. 85, pp. 1071-1082, 2012. 


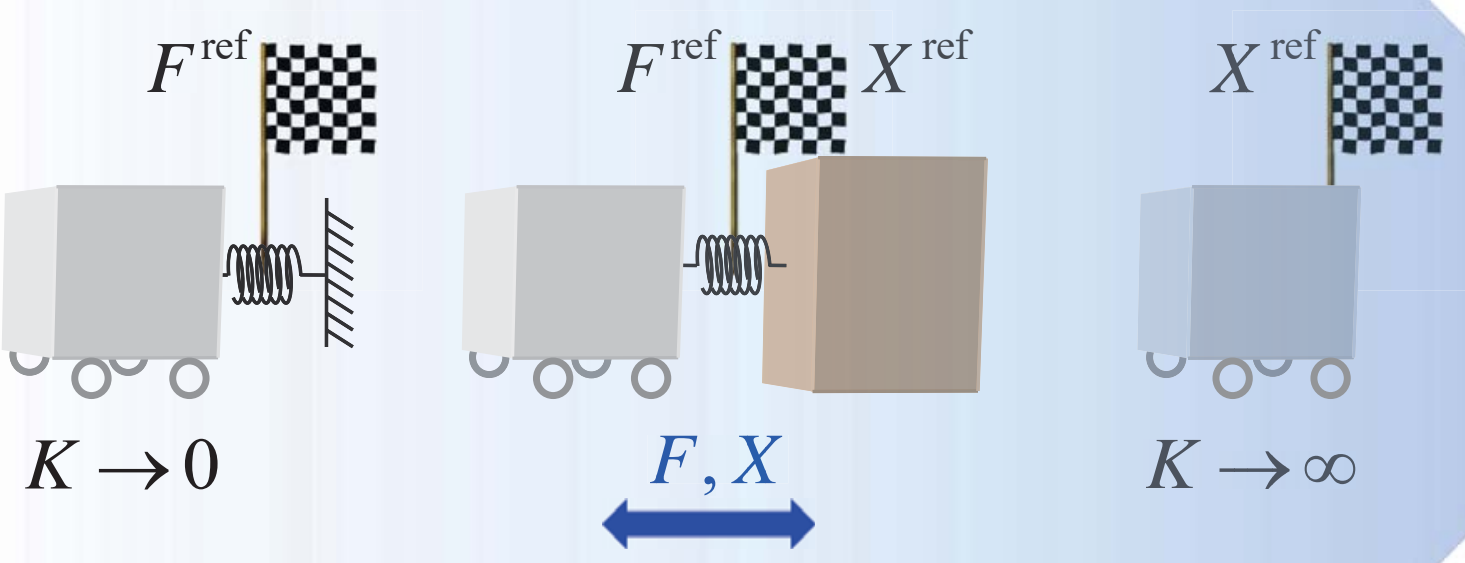




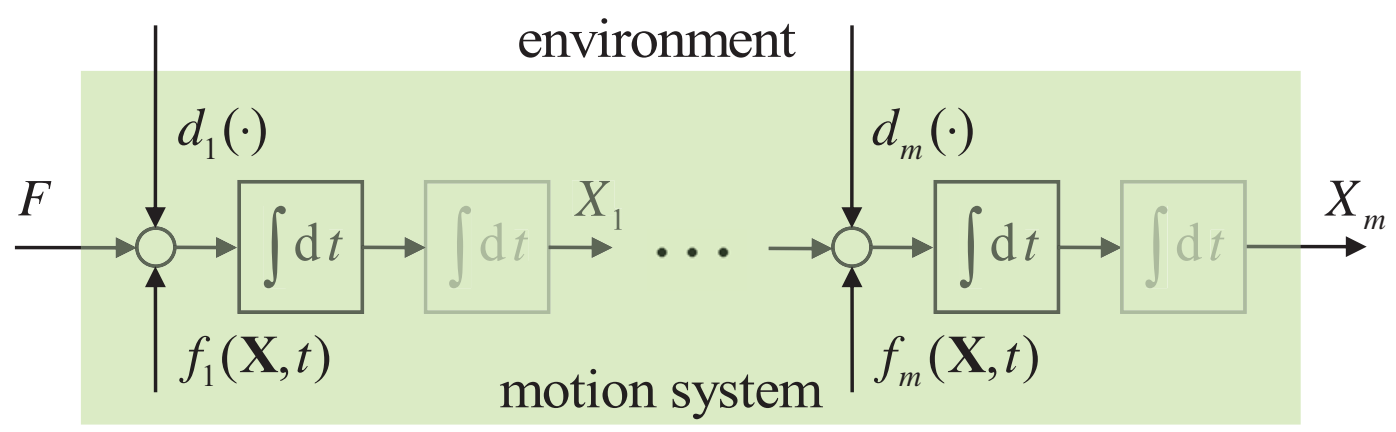




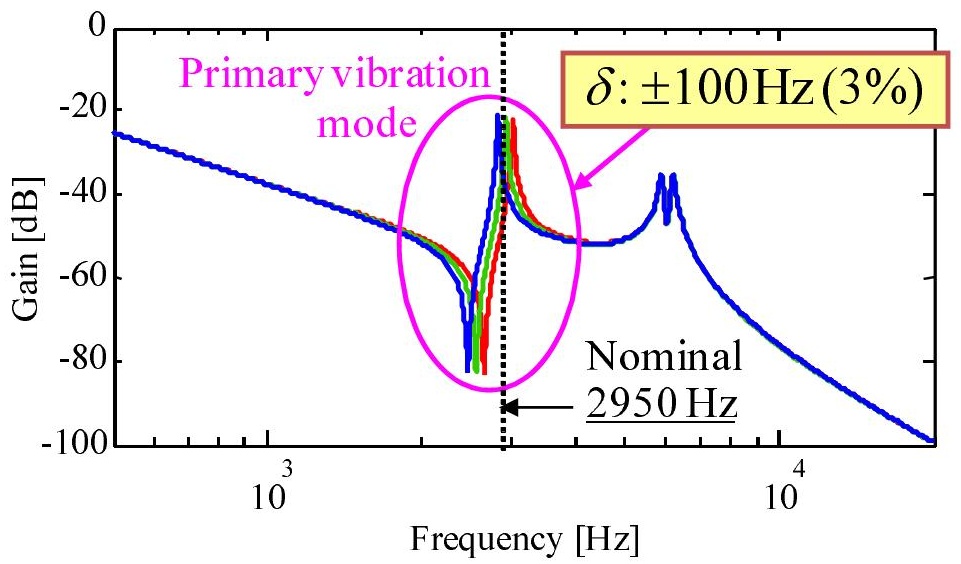




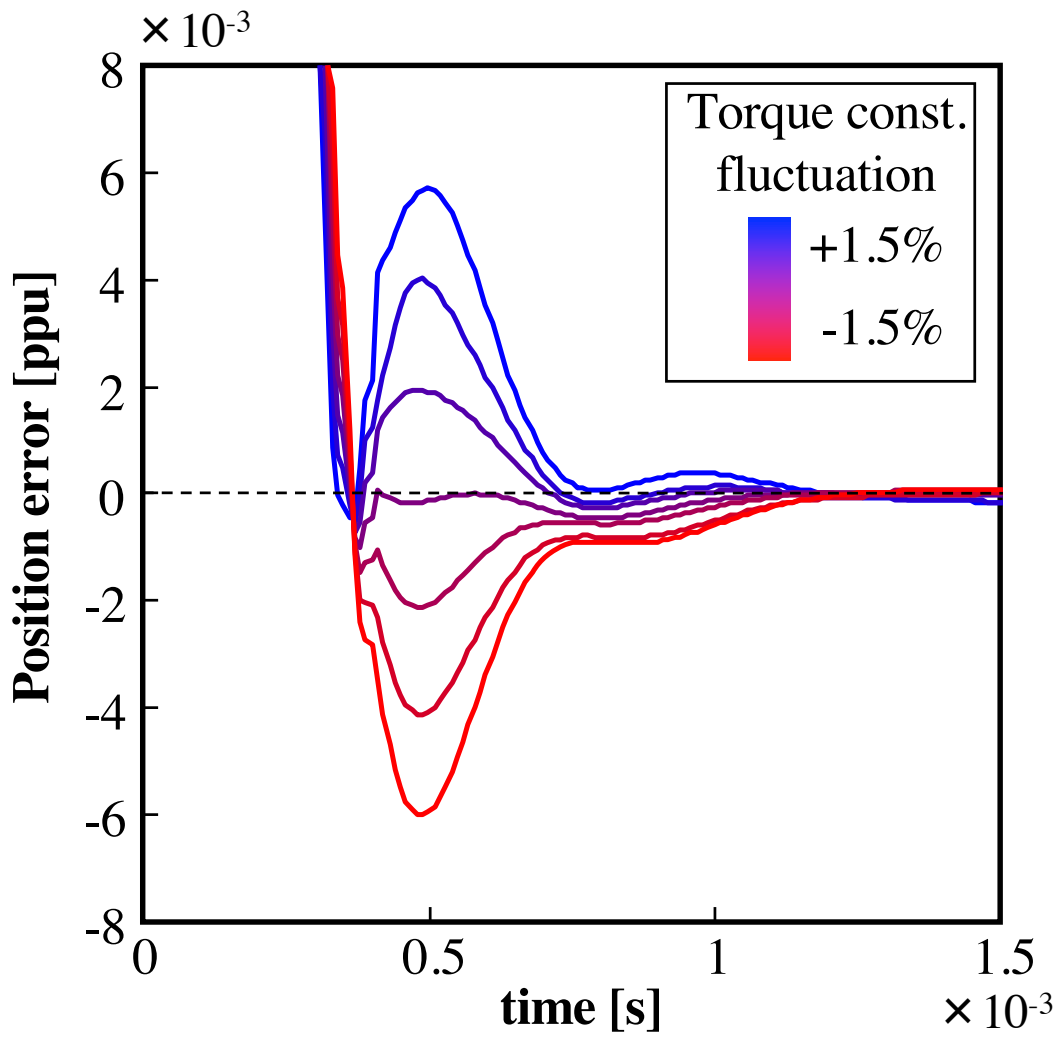




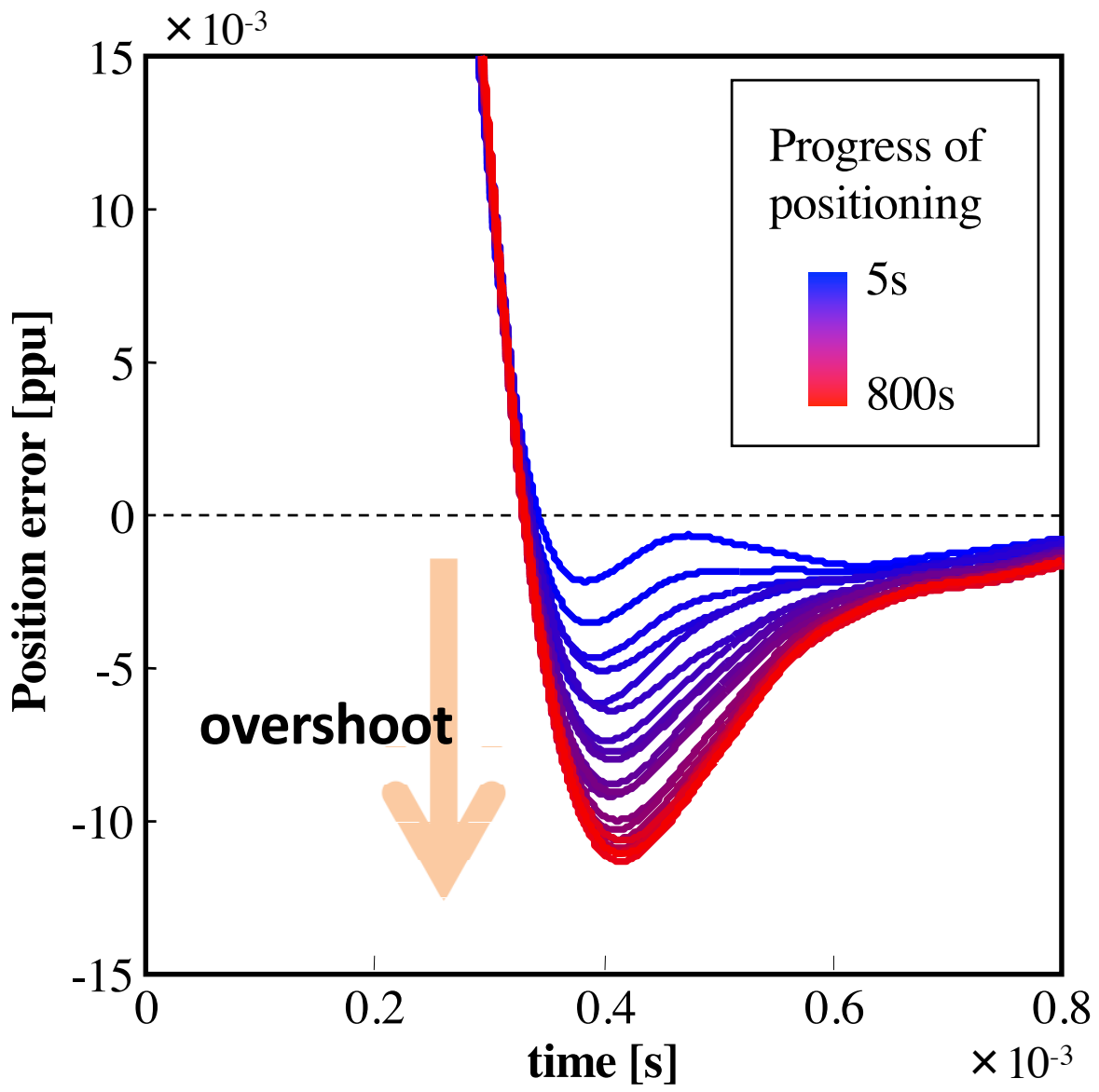


(a)

(b)

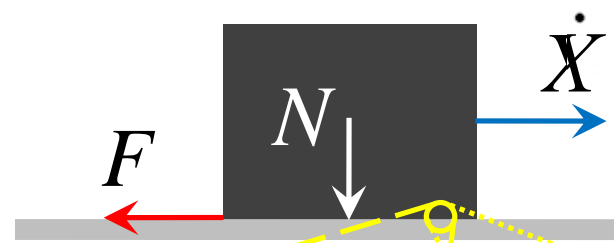
deformable upper surface

(c)
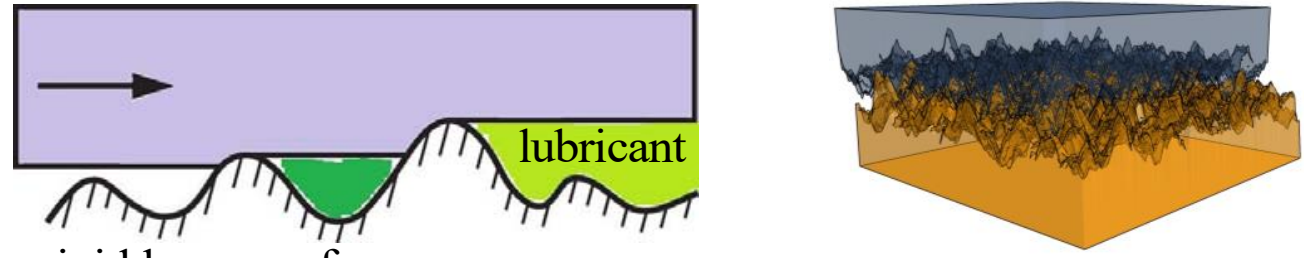
rigid lower surface 


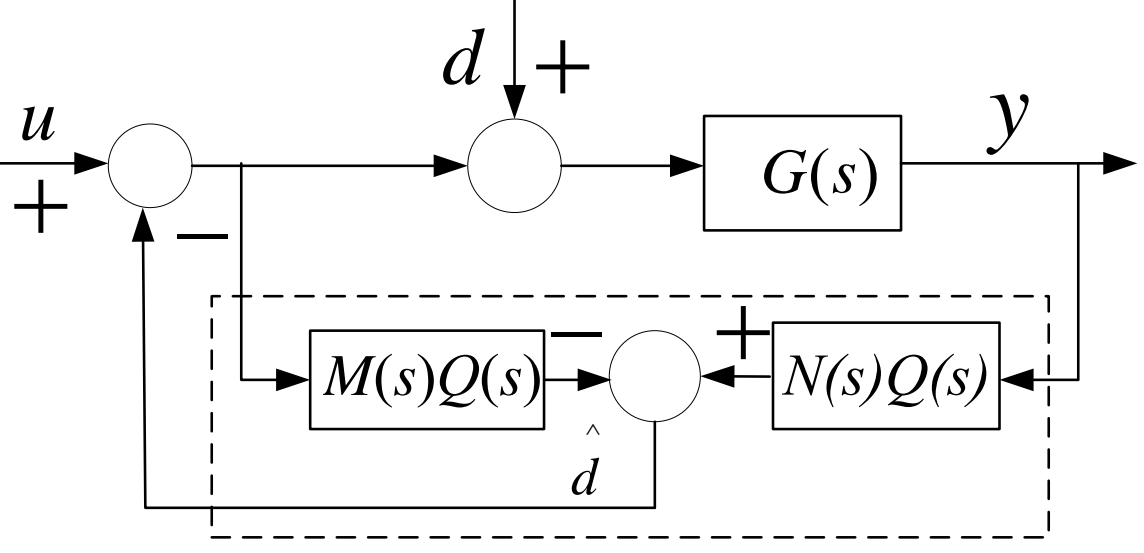

